University of Wollongong

Research Online

Faculty of Engineering and Information

Faculty of Engineering and Information

Sciences - Papers: Part A

Sciences

$1-1-2013$

\title{
Efficient and dynamic key management for multiple identities in identity- based systems
}

Hua Guo

University of New Mexico, Beihang University

Chang Xu

Beihang University

Zhoujun Li

National University of Defense Technology, Beihang University

Yanqing Yao

Beihang University

$\mathrm{Yi} \mathrm{Mu}$

University of Wollongong, ymu@uow.edu.au

Follow this and additional works at: https://ro.uow.edu.au/eispapers

Part of the Engineering Commons, and the Science and Technology Studies Commons

Research Online is the open access institutional repository for the University of Wollongong. For further information contact the UOW Library: research-pubs@uow.edu.au 


\title{
Efficient and dynamic key management for multiple identities in identity-based systems
}

\begin{abstract}
The traditional identity-based cryptography requires a user, who holds multiple identities, to hold multiple private keys, where each private key is associated with an identity. Managing multiple private/public keys is a heavy burden to a user due to key management and storage. The recent advancement of identitybased cryptography allow a single private key to map multiple public keys (identities); therefore the private key management is simplified. Unfortunately, the existing schemes capturing this feature do not allow dynamic changes of identities and have a large data size proportional to the number of the associated identities. To overcome these problems, in this paper, we present an efficient and dynamic identity-based key exchange protocol and prove its security under the Bilinear Diffie-Hellman assumption in the random oracle model. Our protocol requires a relatively small bandwidth for a key agreement communication, in comparison with other existing schemes. (C) 2012 Elsevier Inc. All rights reserved.
\end{abstract}

\section{Keywords}

dynamic, efficient, systems, key, identity, management, multiple, identities

\section{Disciplines}

Engineering | Science and Technology Studies

\section{Publication Details}

Guo, H., Xu, C., Li, Z., Yao, Y. \& Mu, Y. (2013). Efficient and dynamic key management for multiple identities in identity-based systems. Information Sciences, 221 579-590. 


\title{
Efficient and Dynamic Key Management for Multiple Identities in Identity-based Systems
}

\author{
Hua Guo ${ }^{1}$, Chang $\mathrm{Xu}^{1}$, Yanqing $\mathrm{Yao}^{1}$, Zhoujun $\mathrm{Li}^{1,2}$, Yi Mu \\ ${ }^{1}$ State Key Laboratory of Software Development Environment, Beihang University, Beijing, China \\ ${ }^{2}$ Beijing Key Laboratory of Network Technology, Beihang University, Beijing, China \\ ${ }^{3}$ School of Computer Science Software Engineering, University of Wollongong, NSW, Australia
}

\begin{abstract}
The traditional identity-based cryptography requires a user who holds multiple identities to keep multiple private keys, where each private key is associated with an identity. Managing multiple private/public keys is a heavy burden for a user in terms of key management and storage. The recent advances of identity-based cryptography allow a single private key to map multiple public keys (identities), which simplifies the private key management. Unfortunately, the existing schemes do not allow dynamic changes of identities and have a large data size proportional to the number of the associated identities. To overcome these problems, in this paper, we present an efficient and dynamic identity-based key exchange protocol and prove its security under the Bilinear Diffie-Hellman assumption in the random oracle model. Our protocol requires a relatively small bandwidth for a key agreement communication, in comparison with other existing schemes.
\end{abstract}

Keywords: Identity-based Key Management; Provable Security; Informatics Security.

\section{Introduction}

The notion of identity-based cryptography was introduced by Shamir [15]. The identity-based cryptography exhibits the feature of simplicity in public key management, since an user identity such as an email address can be used as a public key. The private key associated with a user's identity is created by the Private Key Generator (PKG) using its master key. Although identity-based cryptography has exhibited many useful features and many concrete identity-based cryptosystems are proposed [3, 4, 5, 6, 17, 15, 16, 17, 18, an obvious key management issue raises, when a user holds multiple identities used as his public keys, since multiple identities lead to multiple private keys.

Multiple identities are very useful for authenticated key distribution. In order to prevent false identification due to identity theft, in common practice, a client is often required to provide his/her multiple identities such as his/her name, driver's license number, bank account number, etc., for applying for a credit card for instance. While a client wants to access his/her taxation information, he/she needs to provide his/her name, tax number, etc. Such applications are usually asymmetric, namely the client and the server can provide different types of identifications. Managing multiple private/public keys is unfortunately a burden for a user. Moreover, using multiple private keys and public keys in a key agreement protocol could cause a high computational complexity. 
To simplify private key management for a user with multiple identities, we seek a solution that multiple identities can be mapped to a single private key. Guo, $\mathrm{Mu}$, and Chen [10] firstly introduced an encryption scheme that provides a solution of multiple identities. Based on [10], Guo, Mu, Zhang, et al. [12 presented a novel authenticated key agreement protocol. To prove the security of their scheme in the random oracle model, they introduced a new assumption called $n$-multiple bilinear collision attack assumption (n-MBCAA1). Although the solutions due to [10, 12] are elegant, they have a relatively high computational complexity, because the computational cost of the scalar multiplication operations, which were adopted in their schemes, is proportional to the number of identities (say $n$ ). Later, Guo, $\mathrm{Mu}$, Chen and $\mathrm{Xu}$ [11] presented a more efficient encryption scheme in the selective-identity model based on the Decisional Bilinear Diffie-Hellman (DBDH) problem. The scalar multiplications on the elliptic curves in their scheme is still proportional to the number of identities (say $n$ ). Very recently, Guo, et al. [13] presented a new efficient key agreement protocol using matrix theory based on Bilinear Diffie-Hellman (BDH). In their scheme, the number of the scalar multiplications on the elliptic curves is proportional to the number of the selected identities (say, $k(k<n)$ ). None of these schemes offers a low communication cost. Although Guo, et al.'s scheme [13] is the most efficient one in terms of the number of the messages, the number of the messages in their scheme is still proportional to the number of the selected identities $k$.

Apart from the issues of computational complexity, communication complexity and security proofs, we believe that a complete multi-identity scheme should capture the feature of dynamic changes of user identities. Actually, some practical applications require the users to change their identities from time to time. For example, online payment systems may require the user name, birthday, address, phone number and bank account number; online insurance services may require the user name, birthday, phone number and driver's licence; online taxation services may require the user name, phone number and tax number. Dynamic changes of the identities cause the corresponding resultant private key be invalid. A trivial solution could be that when a user wants to change his identities, he asks the PKG to generate a new private key. This might be reasonable if the user changes all of his old identities at the same time. However, it might not be the case in practice, since a user might want to change only some of his identities. If we follow the trivial solution, a complete re-computation process has to be carried out, even if only a single identity needs to be added or removed. It would be desirable if the multi-identity private key can be dynamically updated, in the sense that a new identity can be added or an existing identity can be deleted without the re-computation of the private key initially generated by the PKG. 1 .

Practical applications usually require some common basic identities, such as user name, birthday, phone number, address, etc. If all of these basic identities are created as a credential, then a user who has these basic identities can keep one private key corresponding to multiple identities. When a new identity is involved, PKG only needs to authenticate this new identity and compute a private key corresponding to this new identity. Furthermore, a new resultant private key is computed by the user using credential and the new private key from PKG. For example, when a client wants to connect to his/her bank account, he/she asks PKG to generate a private key corresponding to his/her account number. Since he/she can compute the resultant private key related to the basic identities and the

\footnotetext{
${ }^{1}$ It seems that there is no motivation for the user to delete an identity, because even if it doesnt delete an identity, it still can establish session keys with other users by following the protocol. Therefore we only considered the scenario of adding a new identity in this paper.
} 
account number, his/her bank can use the client's basic identities plus the account number to establish a shared key with the client. When a client wants to connect to his/her online insurance services, he/she asks PKG to generate a private key corresponding to his/her driver's licence.

Based on the above observation, we present an efficient dynamic identity-based key management protocol in this paper. Our scheme exhibits the following features:

- A user can dynamically update his private key.

- Our scheme is efficient in terms of the communication cost.

- The security of our scheme can be reduced to the Bilinear Diffie-Hellman assumption.

All the existing schemes do not naturally accommodate the feature of dynamical private-key update. However, we observe that the scheme in [11 implies a dynamical key update feature, but it is conditional, i.e., it requires the PKG to save random numbers used in the private key setup phase for each user. Since a PKG could serve many users, this requirement introduces an additional key management workload for the PKG. This requirement also contradicts the traditional requirement to a PKG, where it does not need to save any parameters for a privacy key generation. Note that although the scheme in [13] allows a user's identities change dynamically, the user's private key is unchanged. This does not meet our "dynamically key management" requirement. The other drawback of the existing schemes is the computation and communication cost, in that the cost is proportional to the number of all identities used for the private key computation or the number of the selected identities.

The rest of this paper is organized as follows. In Section 2, we describe the preliminaries including bilinear pairing, security assumption and security model. In Section 3, we analyze three kinds of key extract algorithms and explain which one can be used to construct dynamical private keys. In Section 4, we present a dynamic multi-identity key management protocol and compare our scheme with all known schemes in terms of computation, communication and dynamic property. In Section 5 , we prove the security of the multi-identity key management protocol. In Section 6, we conclude the paper.

\section{Preliminaries}

In this section, we introduce the background knowledge that will be used for our scheme. We give the basic definition and properties of bilinear pairings, the computational problems and the security model.

\subsection{Bilinear Map}

We first revisit the basic definition of bilinear map and the Bilinear Diffie-Hellman problem. The detail can be found in [4].

The bilinear map $\hat{e}$ is defined over two groups of the same prime-order $q$ denoted by $\mathbb{G}$ and $\mathbb{G}_{T}$ in which the Computational Diffie-Hellman problem is hard. Formally, we have the following definition:

Definition 1 (Bilinear Map) Let $\mathbb{G}$ is an additive group of prime order $q$ and $\mathbb{G}_{T}$ a multiplicative group of the same order. Let $P$ denote a generator of $\mathbb{G}$. An admissible pairing is a bilinear map $\hat{e}: \mathbb{G} \times \mathbb{G} \rightarrow \mathbb{G}_{T}$ which has the following properties: 
- Bilinear: given $Q, R \in \mathbb{G}$ and $a, b \in \mathbb{Z}_{q}^{*}$, we have $\hat{e}(a Q, b R)=\hat{e}(Q, R)^{a b}$.

- Non-degenerate: $\hat{e}(P, P) \neq 1_{\mathbb{G}_{T}}$.

- Computable: $\hat{e}$ is efficiently computable.

Typically, the map $\hat{e}$ can be derived from either the Weil pairing or Tate pairing on an elliptic curve over a finite field. More details on how these groups, pairings and other parameters should be selected in practice for efficiency and security can be found in [4, 9, 14].

\subsection{Computational Problems}

Bilinear map captures an important cryptographic problem, i.e., the Bilinear Diffie-Hellman (BDH) problem, which was introduced by Boneh and Franklin [4].

Definition 2 (BDH) Let $\mathbb{G}$ and $\mathbb{G}_{T}$ be two groups of a prime order $q \geq 2^{k}$, where $k$ is security parameter. Let $P \in \mathbb{G}^{*}$ be a generator of $\mathbb{G}$. Suppose that there exists a bilinear map $\hat{e}: \mathbb{G} \times \mathbb{G} \rightarrow \mathbb{G}_{T}$. Let $\mathcal{A}^{B D H}$ be an attacker modeled as a probabilistic Turing machine taking the security parameter $k$ as input. Suppose that $a, b$ and $c$ are uniformly chosen at random from $\mathbb{Z}_{q}^{*}$ and $a P, b P$ and $c P$ are computed. $\mathcal{A}^{B D H}$ is to solve the following problem: Given $\left(\mathbb{G}, \mathbb{G}_{T}, q, \hat{e}, P, a P, b P, c P\right)$, compute $\hat{e}(P, P)^{a b c}$. We define $\mathcal{A}^{B D H}$ 's success probability as

$$
\operatorname{Succ}_{\mathbb{G}, \mathcal{A}^{B D H}}^{B D H}(k)=\operatorname{Pr}\left[\mathcal{A}^{B D H} \text { outputs } \hat{e}(P, P)^{a b c}\right] .
$$

We denote by $\operatorname{Succ}_{\mathbb{G}}^{B D H}\left(t_{B D H}\right)$ the maximal success probability $\operatorname{Succ}_{\mathbb{G}, A^{B D H}}^{B D H}(k)$ over all attackers having running time bounded by $t_{B D H}$ which is polynomial in the security parameter $k$.

Definition 3 (negligible function) A real-valued function $f(l)$ is negligible if for any polynomial $p(l)$, there exists a positive integer $c \in \mathbb{N}$ such that when $\forall l>c,|f(l)|<\frac{1}{p(l)}$ always hold.

The BDH problem is said to be computationally intractable if $\operatorname{Succ}_{\mathbb{G}}^{B D H}\left(t_{B D H}\right)$ is negligible in $k$.

\subsection{Security Models}

In this paper, we will analyze the security of our protocols using a security model which was initially proposed by Bellare and Rogaway in [1] and extended to the public key setting by Blake-Wilson et al. in [2], and later extended to the identity-based key agreement by Chen et al. [7]. The model includes a set of parties and each party involved in a session is modeled by an oracle. An oracle $\Pi_{i, j}^{s}$ denotes an instance of a party $i$ involved with a partner party $j$ in a session $s$ where the instance of the party $j$ is $\Pi_{j, i}^{t}$ for some $t$. These parties cannot communicate directly; instead they only communicate with each other via an adversary. An adversary can access the oracle by issuing some specified queries as follows.

Send $\left(\Pi_{i, j}^{s}, m\right)$ : This query models an active attack. $\Pi_{i, j}^{s}$ executes the protocol and responds with an outgoing message $x$ or a decision to indicate accepting or rejecting the session. If the oracle $\Pi_{i, j}^{s}$ does not exist, it will be created. Note that if $m=\lambda$, then the oracle is generated as an initiator; otherwise as a responder. 
Reveal $\left(\Pi_{i, j}^{s}\right): \Pi_{i, j}^{s}$ returns the session key as its response if the oracle accepts. Otherwise, it returns $\perp$. Such an oracle is called opened.

Corrupt $(i)$ : The party $i$ responds with its private key.

Test $\left(\Pi_{i, j}^{s}\right)$ : At some point, the adversary can make a Test query to a fresh oracle $\Pi_{i, j}^{s}$. $\Pi_{i, j}^{s}$, as a challenger, randomly chooses $b \in\{0,1\}$ and responds with the real agreed session key, if $b=0$; otherwise it returns a random sample generated according to the distribution of the session key.

The security of a protocol is defined using the two-phase game $\mathcal{G}$ played between a malicious adversary $\mathcal{A}$ and a collection of oracles. At the first stage, $\mathcal{A}$ is able to send the above first three oracle queries at will. Then, at some point, $\mathcal{A}$ will choose a fresh session $\Pi_{i, j}^{s}$ on which to be tested and send a Test query to the fresh oracle associated with the test session. After this point, the adversary can continue querying the oracles but can not reveal the test oracle or its partner, and cannot corrupt the entity $j$. Eventually, $\mathcal{A}$ terminates the game simulation and outputs a bit $b^{\prime}$ for $b$. We say that $\mathcal{A}$ wins if the adversary guesses the correct $b$.

Define the advantage of $\mathcal{A}$ as:

$$
\operatorname{Adv}^{\mathcal{A}}(k)=\left|2 \operatorname{Pr}\left[b^{\prime}=b\right]-1\right|,
$$

where $k$ is a security parameter.

The fresh oracle in the game is defined as follows.

Definition 4 (Fresh oracle [8]) An oracle $\Pi_{i, j}^{s}$ is called fresh if (1) $\Pi_{i, j}^{s}$ has accepted; (2) $\Pi_{i, j}^{s}$ is unopened; (3) $j \neq i$ is not corrupted; (4) there is no opened oracle $\Pi_{j, i}^{t}$, which has had a matching conversation to $\Pi_{i, j}^{s}$.

In this work, we use the concatenation of the messages in a session to define the session ID, thus to define the matching conversation, i.e., two oracles $\Pi_{i, j}^{s}$ and $\Pi_{j, i}^{t}$ have a matching conversation to each other if both of them have the same session ID.

A secure authenticated key agreement protocol is defined as follows.

Definition 5 Protocol $\Pi$ is a secure authenticated key agreement protocol, if:

- In presence of the benign adversary (who faithfully relays messages between parties), on $\Pi_{i, j}^{s}$ and $\Pi_{j, i}^{t}$, both oracles always accept holding the same session key and this key is distributed uniformly at random on session key space;

- For every probability polynomial time $(P P T)$ adversary $\mathcal{A}, \operatorname{Adv}^{\mathcal{A}}(k)$ is negligible.

\section{Pairing-based Key Extract Algorithms}

It is necessary to revisit key extraction methods and highlight their applications in key agreement, since key extraction plays an important role in our protocol.

Chen, Cheng and Smart [7] pointed out two types of pairing-based key extract algorithms that have been used in identity-based key agreement schemes. We notice that there is another one used in 
encryption schemes as pointed out by Boyen [5]. We call them "Exponent Inversion Extract", "Commutative Blinding Extract" and "Full Domain Hash Extract", respectively. However, we observed that only the last one can be used to construct a dynamical key extract algorithm.

Exponent Inversion Extract. This algorithm was first introduced by Sakai and Kasahara [16]. Given the pairing parameters, an identity string $I D_{A}$ for a user $A$, a hash-function $H_{1}:\{0,1\} \rightarrow \mathbb{Z}_{q}$, the master private key $s \in \mathbb{Z}_{q}$, and the master public key $R=s P \in \mathbb{G}$, the algorithm computes $\alpha=H_{1}\left(I D_{A}\right) \in \mathbb{Z}_{q}$ and $d_{A}=\frac{1}{s+\alpha} P \in \mathbb{G}$. The values $T_{A}=\alpha P+R=(s+\alpha) P \in \mathbb{G}$ and $d_{A}$ will be used as the public and private key pair respectively corresponding to $A$ 's identity $I D_{A}$. The value $s$ is kept secret by the PKG, and the master public key $R$ is made available to every user.

The encryption scheme given in [10] and the key agreement protocol presented in [12] are based on this key extract algorithm. In both schemes, the corresponding private key for user $A$ is $d_{A}=$ $\frac{1}{\prod_{i=1}^{n}\left(s+\alpha_{i}\right)} P \in \mathbb{G}$ according to the above definition. Since the inverse of the sum of the master key $s$ and the hash value $\alpha_{i}$ of identity $I D_{A, i}$ is the coefficient of the public parameter $P$, it is hard for a user to dynamically compute a private key by himself.

Note that if we construct $d_{A}=\left(\sum_{i=1}^{n} \frac{1}{s+\alpha_{i}}\right) P \in \mathbb{G}$, the user can generate a new private key $d_{A}^{\prime}$. However, the message $T$ generated by the intended recipient $B$ should contain user $A$ 's identities and meanwhile satisfy $\hat{e}\left(d_{A}^{\prime}, T\right)=\hat{e}(P, P)^{r}$ where $r$ is the random value chosen by user $B$. Thus $T$ should have the form $\frac{1}{\sum_{i=1}^{n} \frac{1}{s+\alpha_{i}}} P \in \mathbb{G}$, which is hard for user $B$ to compute due to the hardness of the discrete logarithm on ellipse curves. Therefore this kind of key extract algorithm can not be used to construct dynamical key extract algorithm.

Commutative Blinding Extract. This algorithm was first proposed by Boneh-Boyen [3] to construct identity-based encrypt scheme without random oracle. Given the pairing parameters, an identity string $I D_{A} \in \mathbb{Z}_{p}$ for a user $A$, the master public key $<g, g_{1}, g_{2}, h_{1}>$ where $g$ is a random generator in $\mathbb{G}^{*}, g_{1}=g^{\alpha}$ where $\alpha \in \mathbb{Z}_{p}, g_{2} \in R$ the algorithm computes $d_{A}=\left(g_{2}^{\alpha} \cdot\left(g_{1}^{I D_{A}} h_{1}\right)^{r}, g^{r}\right)$.

The scheme given in [1] is based on this key extract algorithm. The private key in this scheme has the form $d_{I D_{i}}=\left(d_{1}, d_{2}\right)=\left(g_{2}^{\alpha}\left(h k_{1}^{I D_{1}} \cdots k_{i}^{I D_{i}} k_{n}^{I D_{n}}\right)^{r}, g^{r}\right)$, where $k_{i}$ are public and $r$ is generated by PKG. However, this scheme is only conditionally-dynamic due to the structure of this kind of key extract algorithm. More precisely, if a user wants to add a new identity, whether he can compute a new private key depends on whether PKG can remember the same random value for the same user. If $\mathrm{PKG}$ uses the same random value to compute the corresponding private keys for the new identities from a user, then this user can update his private key dynamically. However, the disadvantage is that PKG has to remember many pairs $\left(I D_{i}, r_{I D_{i}}\right)$ for all users. Therefore, this kind of key extract algorithm cannot be used to construct dynamical key extract algorithm.

Full Domain Hash Extract. This algorithm was first proposed by Sakai, Ohgishi and Kasahara [17. Given the pairing parameters $<q, \hat{e}, \mathbb{G}, \mathbb{G}_{T}>$ where $\hat{e}: \mathbb{G} \times \mathbb{G} \rightarrow \mathbb{G}_{T}$, an identity string $I D_{A}$ for a user $A$, a hash-function $H_{1}:\{0,1\}^{*} \rightarrow \mathbb{G}$, the master private key $s \in_{R} \mathbb{Z}_{q}^{*}$, and the master public key $R=s P \in \mathbb{G}$ where $P$ is the generator of $\mathbb{G}$. The algorithm computes $Q_{A}=H_{1}\left(I D_{A}\right) \in \mathbb{G}$ and $d_{A}=s Q_{A} \in \mathbb{G}$. The values $Q_{A}$ and $d_{A}$ will be used as the public and private key pair corresponding to $A$ 's identity $I D$.

In this case, the corresponding private key for user $A$ should be $d_{A}=\sum_{i=1}^{n} s Q_{A, i} \in \mathbb{G}$ according to the above definition. Since the master key $s$ is the coefficient of the hash value of identity $I D_{A, i}$, 
user $A$ can compute the new private key dynamically by himself. Therefore, in this paper we adopt this kind of derivation algorithm.

\section{A Dynamic Multi-identity key management Protocol}

In this section, we present a dynamic multi-identity key exchange protocol. Suppose two parties $A$ and $B$ want to establish a shared session key using this protocol. $A$ holds $m$ IDs $\left(I D_{A, 1}, I D_{A, 2}, \cdots\right.$, $\left.I D_{A, m}\right)$ and $B$ holds $n$ IDs $\left(I D_{B, 1}, I D_{B, 2}, \cdots, I D_{B, n}\right)$.

\subsection{The Scheme}

Our scheme consists of three algorithms: Setup, Extract and Key Agreement.

- Setup: On input a security parameter $\ell$, the PKG chooses a cyclic additive group $\mathbb{G}$ and a cyclic multiplicative group $\mathbb{G}_{T}$ with prime order $q$, where $\mathbb{G}$ is generated by $P$ and there exists a bilinear map ê $: \mathbb{G} \times \mathbb{G} \rightarrow \mathbb{G}_{T}$. The PKG chooses a random $s \in \mathbb{Z}_{q}^{*}$ as the master secret and sets $P_{\text {pub }}=s P$. The PKG also chooses a random $Q \in \mathbb{G}$ and chooses cryptographic hash function $H_{1}:\{0,1\}^{*} \rightarrow \mathbb{G}$. The system parameter list is params $=\left(\mathbb{G}, \mathbb{G}_{T}, \hat{e}, P, P_{p u b}, Q, H_{1}\right)$.

- Extract: Given user ${ }_{i}$ identity tuple $\left(I D_{i, 1}, \cdots, I D_{i, n}\right)$, this algorithm takes as input mastersecret $s$ and generates the private key as follows:

- Compute $P_{i, u}=H_{1}\left(I D_{i, u}\right)$ where $1 \leq u \leq n$.

- Choose a random $r_{i, u} \in \mathbb{Z}_{q}^{*}$ and computes $R_{i, u}^{\prime}=r_{i, u} P$.

- Compute $S_{i, u}^{\prime}=r_{i, u} Q+s P_{i, u}$.

- Compute $S_{i}=\sum_{u=1}^{n} S_{i, u}^{\prime}$ and $R_{i}=\sum_{u=1}^{n} R_{i, u}^{\prime}$.

- Send the private key $d_{i}=\left(S_{i}, R_{i}\right)$ to user $_{i}$ through an authenticated and private channel.

- Key Agreement: Suppose user $A$ is an initiator and has $m$ identities, $B$ is an intended partner and has $n$ identities. Suppose $A$ and $B$ are in the different domain, then they should have different system parameters, i.e., params Ident $=\left(\mathbb{G}, \mathbb{G}_{T}, \hat{e}, P, P_{\text {pub }}^{\text {Ident }}, Q^{\text {Ident }}, H_{1}^{\text {Ident }}\right)$ where Ident $=\{A, B\}$ and $P_{\text {pub }}^{\text {Ident }}=s^{\text {Ident }} P$. Note that $\mathbb{G}, \mathbb{G}_{T}, \hat{e}, P$ can be selected as the same from the standard parameters sets, while $s^{\text {Ident }}, Q^{\text {Ident }}, H_{1}^{\text {Ident }}$ are selected by users' PKG. $A$ and $B$ select a hash function $H_{2}:\{0,1\}^{*} \rightarrow\{0,1\}^{r}$, and they have the private key as $d_{A}$ and $d_{B}$, respectively.

Let $P_{A, j}=H_{1}^{A}\left(I D_{A, j}\right), P_{B, j}=H_{1}^{B}\left(I D_{B, j}\right) . A$ wants to use $k^{\prime}$ identities $\left(I D_{B, 1}, \cdots, I D_{B, k^{\prime}}\right)$ from $B$ 's identity set where $k^{\prime} \leq n$. Similarly, $B$ wants to use $k$ identities $\left(I D_{A, 1}, \cdots, I D_{A, k}\right)$ from $A$ 's identity set where $k \leq m$.

To establish a shared session secret key, $A$ and $B$ conduct the following tasks:

- $A$ selects a random value $x \in \mathbb{Z}_{q}^{*}$, and computes $T_{A, 1}=x P, T_{A, 2}=-x Q^{B}$ and $T_{A, 3}=$ $x \sum_{j=k^{\prime}+1}^{n} P_{B, j} . A$ sends $T_{A}=\left(T_{A, 1}, T_{A, 2}, T_{A, 3}\right)$ to user $B$.

- Upon receiving a message $T_{A}, B$ selects a random value $y \in \mathbb{Z}_{q}^{*}$, and computes $T_{B, 1}=y P$, $T_{B, 2}=-y Q^{A}$ and $T_{B, 3}=y \sum_{j=k+1}^{m} P_{A, j} . B$ sends $T_{B}=\left(T_{B, 1}, T_{B, 2}, T_{B, 3}\right)$ to user $A$. 
After the exchange, $A$ computes the session secrets as

$$
K_{A B, 1}=\hat{e}\left(S_{A}, T_{B, 1}\right) \cdot \hat{e}\left(T_{B, 2}, R_{A}\right) \cdot \hat{e}\left(T_{B, 3},-P_{p u b}^{A}\right) \cdot \hat{e}\left(\sum_{j=1}^{k^{\prime}} P_{B, j}, P_{p u b}^{B}\right)^{x} .
$$

and

$$
K_{A B, 2}=x \cdot T_{B, 1}=x y P .
$$

Similarly, $B$ computes the session secret as

$$
K_{B A, 1}=\hat{e}\left(S_{B}, T_{A, 1}\right) \cdot \hat{e}\left(T_{A, 2}, R_{B}\right) \cdot \hat{e}\left(T_{A, 3},-P_{p u b}^{B}\right) \cdot \hat{e}\left(\sum_{j=1}^{k} P_{A, j}, P_{p u b}^{A}\right)^{y} .
$$

and

$$
K_{B A, 2}=y \cdot T_{A, 1}=x y P .
$$

Remark 1 It is very easy for a user to compute a new private key when he adds new identities. For example, when user $A$ adds a new identity $I D_{A, m+1}$ to his identity set, he asks a new private key corresponding to this new identity from PKG. PKG authenticates this new identity and generates a private key $d_{A, m+1}=\left(S_{A, m+1}^{\prime}, R_{A, m+1}^{\prime}\right)$ where $S_{A, m+1}^{\prime}=r_{A, m+1} Q^{A}+s^{A} P_{A, m+1}$ and $R_{A, m+1}^{\prime}=$ $r_{A, m+1} P$. Once receives the private key $d_{A, m+1}, A$ can aggregates this private key with the old one to compute a new private key $d_{A}^{\prime}=\left(S_{A}^{\prime}, R_{A}^{\prime}\right)$ as follows:

$$
\begin{gathered}
S_{A}^{\prime}=S_{A}+S_{A, m+1}^{\prime}=\sum_{j=1}^{m} S_{A, j}^{\prime}+S_{A, m+1}^{\prime}=\sum_{j=1}^{m+1} S_{A, j}^{\prime}, \\
R_{A}^{\prime}=R_{A}+R_{A, m+1}^{\prime}=\sum_{j=1}^{m} R_{A, j}^{\prime}+R_{A, m+1}^{\prime}=\sum_{j=1}^{m+1} R_{A, j}^{\prime} .
\end{gathered}
$$

\subsection{Correctness}

We verify the correctness of the protocol. Take user $A$ as an example.

$$
\begin{aligned}
K_{A B, 1}= & \hat{e}\left(S_{A}, T_{B, 1}\right) \cdot \hat{e}\left(T_{B, 2}, R_{A}\right) \cdot \hat{e}\left(T_{B, 3},-P_{p u b}^{A}\right) \cdot \hat{e}\left(\sum_{j=1}^{k^{\prime}} P_{B, j}, P_{p u b}^{B}\right)^{x} \\
= & \hat{e}\left(\sum_{j=1}^{m} s^{A} P_{A, j}, y P\right) \cdot \hat{e}\left(\sum_{j=1}^{m} r_{A, j} Q^{A}, y P\right) \cdot \hat{e}\left(-y Q^{A}, \sum_{j=1}^{m} r_{A, j} P\right) \cdot \hat{e}\left(y \sum_{j=k+1}^{m} P_{A, j},-s^{A} P\right) \\
& \cdot \hat{e}\left(\sum_{j=1}^{k^{\prime}} P_{B, j}, P_{p u b}^{B}\right)^{x} \\
= & \hat{e}\left(\sum_{j=1}^{k} s^{A} P_{A, j}, y P\right) \cdot \hat{e}\left(\sum_{j=k+1}^{m} s^{A} P_{A, j}, y P\right) \cdot \hat{e}\left(y \sum_{j=k+1}^{m} P_{A, j},-s^{A} P\right) \cdot \hat{e}\left(\sum_{j=1}^{k^{\prime}} P_{B, j}, s^{B} P\right)^{x} \\
= & \hat{e}\left(\sum_{j=1}^{k} P_{A, j}, s^{A} P\right)^{y} \cdot \hat{e}\left(\sum_{j=1}^{k^{\prime}} P_{B, j}, P_{p u b}^{B}\right)^{x} \\
= & \hat{e}\left(\sum_{j=1}^{k} P_{A, j}, P_{p u b}^{A}\right)^{y} \cdot \hat{e}\left(\sum_{j=1}^{k^{\prime}} P_{B, j}, P_{p u b}^{B}\right)^{x} .
\end{aligned}
$$


Similarly, $B$ computes the session key as

$$
\begin{aligned}
K_{B A, 1} & =\hat{e}\left(S_{B}, T_{A, 1}\right) \cdot \hat{e}\left(T_{A, 2}, R_{B}\right) \cdot \hat{e}\left(T_{A, 3},-P_{p u b}^{B}\right) \cdot \hat{e}\left(\sum_{j=1}^{k} P_{A, j}, P_{p u b}^{A}\right)^{y} \\
& =\hat{e}\left(\sum_{j=1}^{k} P_{A, j}, P_{p u b}^{A}\right)^{y} \cdot \hat{e}\left(\sum_{j=1}^{k^{\prime}} P_{B, j}, P_{p u b}^{B}\right)^{x} .
\end{aligned}
$$

Thus, the two secret keys computed by $A$ and $B$ are equal and $A$ and $B$ have successfully established the shared key $K_{1}=K_{A B, 1}=K_{B A, 1}$ and $K_{2}=K_{A B, 2}=K_{B A, 2}$ after running an instance of the protocol. The final shared session key is then $s k=H_{2}\left(A\|B\| T_{A}\left\|T_{B}\right\| K_{1} \| K_{2}\right)$.

\subsection{Efficiency Analysis and Comparison}

We compare our scheme with other existing schemes. Note that Scheme 2 [12] and Scheme 4 [13] are key agreement schemes, while Schemes 1 [10] and Scheme 3 [11] are actually encryption schemes, which can be used as key agreement schemes run by two users. Suppose user $A$ has $m$ identities and $k$ selected identities.

\begin{tabular}{|c|c|c|c|c|}
\hline \hline Schemes & Computation $(\mathrm{A}+\mathrm{M}+\mathrm{P})$ & Communication & Dynamic & Security Reduction \\
\hline Scheme $1[10]$ & $O(m)+O(m)+2$ & $m+2$ & no & q-BSDH \\
\hline Scheme 2 [12] & $O(m)+O(m)+2$ & $m+1$ & no & $k$-MBCAA1 \\
\hline Scheme 3 [11] & $1+m+3$ & $m+2$ & conditional & DBDH \\
\hline Scheme 4 [13] & $O(k)+O(k)+3$ & $k$ & yes & BDH \\
\hline Ours & $O(m)+4+4$ & 3 & yes & BDH \\
\hline
\end{tabular}

Table 1: The comparison of the proposed scheme and other schemes. "A" denotes addition operation on $\mathbb{G}$, "M" denotes scalar multiplication operation on $\mathbb{G}$, and "P" denotes pairing operation, respectively. ${ }^{*}$ Scheme 4 allows the user to change its identities dynamically, while keeps the private key unchanged.

From table1, we can find that our protocol is more efficient than Scheme 1, Scheme 2 and Scheme 4. We observe that Scheme 3 needs only one addition operation while our scheme needs $O(m)$. However, Scheme 3 needs $m$ scalar multiplication operations which are much more expensive than the addition operation. Therefore, our scheme is more efficient in comparison to Scheme 3, if $m>4$ in terms of the computation cost. In terms of the communication cost, only our scheme offers a constant size of the messages exchanged. Scheme 4 is better than ours only when $k \leq 2$. Thus, considering both computation and communication costs, our scheme exhibits the best efficiency.

Our scheme and Scheme 4 are the only schemes that offer a full dynamical key update feature. Note that Scheme 4 allows the user to change its identities dynamically, while keeping the private key unchanged. In our scheme, the private key changes with the change of the user's identities. We cannot regard Scheme 3 as dynamic, since the PKG has to store the random value for every user for computations of private keys.

From the last column, we can see that Scheme 1 is based on a strong security assumption named Bilinear Strong Diffie-Hellman assumption (q-BSDH for short). Unfortunately, the security proof for 
Scheme 1 is pointed out to be incorrect [12. The security for Scheme 2 relies on a new assumption called $k$-multiple bilinear collision attack assumption ( $k$-MBCAA1 for short) which is proved to be equivalent to the known $k$-bilinear collision attack assumption. However this security assumption is too strong. Scheme 3 is based on the Decisional Bilinear Diffie-Hellman (DBDH) assumption, while Scheme 4 and our scheme are based on the computational Bilinear Diffie-Hellman (BDH) assumption which is the weakest assumption among all of the above assumptions.

\section{$5 \quad$ Security Analysis}

Theorem 1 If $H_{1}$ and $H_{2}$ are random oracles and the $B D H$ assumption holds, then our dynamic multi-identity scheme is a secure key agreement protocol. Suppose $\mathcal{A}$ is an adversary that attacks the multi-identity scheme in the random oracle model with non-negligible probability $\epsilon$ and makes at most $q_{1}, q_{2}$ queries to $H_{1}$ and $H_{2}$, respectively, and creates at most $q_{o}$ oracles. Then there exists an algorithm $\mathcal{B}$ to solve the $B D H$ problem with advantage

$$
A d v_{\mathcal{B}}^{B D H} \geq \frac{1}{q_{1} \cdot q_{o} \cdot q_{2}} \cdot \epsilon
$$

Proof: For simplicity, we assumed that all users belong to the same PKG. This assumption would not affect the security proof. $2^{2}$

We define Session ID as a concatenation of $T_{A} \| T_{B}$. We focus on how to construct an algorithm $\mathcal{B}$ using the adversary $\mathcal{A}$ to solve a BDH problem with non-negligible probability.

Given an instance of the BDH problem

$$
\left\langle q, k, \mathbb{G}, \mathbb{G}_{T}, \hat{e}, P, a P, b P, c P\right\rangle
$$

where $\hat{e}$ is a bilinear pairing $\hat{e}: \mathbb{G} \times \mathbb{G} \rightarrow \mathbb{G}_{T}, \mathcal{B}$ 's task is computing $\hat{e}(P, P)^{a b c}$.

Setup: $\mathcal{B}$ simulates the Setup algorithm as follows:

$\mathcal{B}$ chooses a random value $y \in \mathbb{Z}_{q}^{*}$ and computes $Q=y P$. Then $\mathcal{B}$ sets $P_{\text {pub }}=a P$ where $a$ is the master key which is unknown to the simulator. After that, $\mathcal{B}$ chooses two hash functions $H_{1}$ and $H_{2}$, and sends the system parameters $\left\langle\mathbb{G}, \mathbb{G}_{T}, \hat{e}, P, Q, P_{p u b}, H_{1}, H_{2}\right\rangle$ to $\mathcal{A}$. The hash functions $H_{1}$ and $H_{2}$ are random oracles controlled by $\mathcal{B}$.

Algorithm $\mathcal{B}$ randomly chooses $I \in_{R}\left\{1, \cdots q_{1}\right\}, J \in_{R}\left\{1, \cdots, q_{o}\right\}$ and $K \in_{R}\{1, \cdots, n\}$ and begins its simulation. Here, we should note that notation $I D_{i, u}$ means the $u$-th chosen identity of user $i$, and $\Pi_{i, j}^{s}$ is the $s$-th oracle among all the created oracles. Also note that $n_{i, j}^{t}$ means the number of the identities $j$ chooses from $i$ 's identities set in this session. For simplicity, we assume that each party has $n$ identities. Algorithm $\mathcal{B}$ answers the queries which are asked by adversary $\mathcal{A}$ in arbitrary order as follows.

$H_{1}\left(I D_{i, u}\right)$ queries: Algorithm $\mathcal{B}$ maintains an initially empty list $H_{1}^{\text {list }}$ with entries of the form $\left(i, I D_{i, u}, h_{i, u}, d_{i, u}, v_{i, u}\right)$. When $\mathcal{A}$ queries the oracle $H_{1}$ at a point $I D_{i, u}, \mathcal{B}$ responds to the query as follows:

\footnotetext{
${ }^{2}$ When users belong to the different domains, the only difference is that the simulator needs to prepare two sets of system parameters $<\mathbb{G}, \mathbb{G}_{T}, \hat{e}, P, Q_{i}, P_{\text {pub }}^{i}, H_{1}^{i}, H_{2}>$ and $<\mathbb{G}, \mathbb{G}_{T}, \hat{e}, P, Q_{i}, P_{\text {pub }}^{j}, H_{1}^{j}, H_{2}>(i, j$ are different users $)$ to the adversary, where only $Q_{i}, P_{p u b}$ and $H_{1}$ are different. Then the simulator can use these corresponding parameters to proceed the proof according to the description of the protocol. Thus this assumption would not affect the security proof.
} 
- If $I D_{i, u}$ already appears on the $H_{1}^{l i s t}$ in a tuple $\left(i, I D_{i, u}, h_{i, u}, d_{i, u}, v_{i, u}\right)$, then $\mathcal{B}$ responds with $H_{1}\left(I D_{i, u}\right)=h_{i, u}$.

- If $I D_{i, u}$ is the $J$-th unique query to $H_{1}$ and $(i, u)=(I, K)$, then $\mathcal{B}$ selects a random value $v_{i, u} \in \mathbb{Z}_{q}^{*}$ and stores $\left(i, I D_{i, u}, b P, \perp, v_{i, u}\right)$ into the tuple list and responds with $H_{1}\left(I D_{i, u}\right)=b P$.

- Otherwise, $\mathcal{B}$ firstly selects $l_{i, u} \in \mathbb{Z}_{q}^{*}$ and $v_{i, u} \in \mathbb{Z}_{q}^{*}$ randomly, then computes $H_{1}\left(I D_{i, u}\right)=h_{i, u}=$ $l_{i, u} P$ and $d_{i, u}=l_{i, u} \cdot a P . \mathcal{B}$ inserts $\left(i, I D_{i, u}, h_{i, u}, d_{i, u}, v_{i, u}\right)$ into the tuple list and responds with $H_{1}\left(I D_{i, u}\right)=h_{i, u}$.

$H_{2}\left(I D_{i}, I D_{j}, T_{i}^{t}, T_{j}^{t}, K_{1}^{t}, Z^{t}\right)$ queries: $\mathcal{B}$ maintains an initially empty list $H_{2}^{\text {list }}$ with entries of the form $\left(I D_{i}, I D_{j}, T_{i}^{t}, T_{j}^{t}, K_{1}^{t}, Z^{t}, \zeta^{t}\right)$ which is indexed by $\left(I D_{i}, I D_{j}, T_{i}^{t}, T_{j}^{t}, K_{1}^{t}, Z^{t}\right)$. $\mathcal{B}$ responds to the query in the following way.

- If a tuple indexed by $\left(I D_{i}, I D_{j}, T_{i}^{t}, T_{j}^{t}, K_{1}^{t}, Z^{t}\right)$ is on the list, then $\mathcal{B}$ responds with $\zeta^{t}$.

- Otherwise, $\mathcal{B}$ goes though the list $\mathcal{L}$ to find a tuple $\left(I D_{i}, I D_{j}, T_{i}^{t}, T_{j}^{t}, \Pi_{i, j}^{t}\right)$ and proceeds as follows.

- Note that since there are three messages in each message tuple $T_{i}^{t}$ and $T_{j}^{t}$, let $m_{k}^{T_{i}^{t}}(k=$ $1,2,3)$ denote the $k$-th message of message tuple $T_{i}^{t}$ and $m_{k}^{T_{j}^{t}}(k=1,2,3)$ the $k$-th message of message tuple $T_{j}^{t}$. Test if $\hat{e}\left(m_{1}^{T_{i}^{t}}, m_{1}^{T_{j}^{t}}\right)=\hat{e}\left(P, Z^{t}\right)$ holds. If the equation holds, $\mathcal{B}$ does the following:

* Find the values $f_{i, j}^{t}$ and $S K_{i, j}^{t}$ corresponding to oracle $\Pi_{i, j}^{t}$ from the list $\Omega$ in the Send query.

* Find the value $l_{j, u}^{t}$ from $H_{1}^{l i s t}$ for party with $I D_{j}$.

* Compute the shared secret via the following equation

$$
\begin{aligned}
K_{i j, 1}^{t}= & \hat{e}\left(S_{i}, m_{1}^{T_{j}^{t}}\right) \cdot \hat{e}\left(m_{2}^{T_{j}^{t}}, R_{i}\right) \cdot \hat{e}\left(m_{3}^{T_{j}^{t}},-P_{p u b}\right) \cdot \hat{e}\left(\sum_{u=1}^{n_{j, i}^{t}} h_{i, u}, a P\right)^{f_{i, j}^{t} a} \\
= & \hat{e}\left(\sum_{u=1, u \neq K}^{n} l_{i, u} a P+v_{i, u} y P, m_{1}^{T_{j}^{t}}\right) \cdot \hat{e}\left(\frac{1}{f_{i, j}^{t}} Z_{t}, b P\right) \cdot \hat{e}\left(m_{2}^{T_{j}^{t}}, \sum_{u=1}^{n} v_{i, u} P\right) \\
& \cdot \hat{e}\left(m_{3}^{T_{j}^{t}},-P_{p u b}\right) \cdot \hat{e}\left(\sum_{j=1}^{n_{j, i}^{t}} l_{i, u} a P, a P\right)^{f_{i, j}^{t}} .
\end{aligned}
$$

Note that $\Pi_{i, j}^{t}$ is put on the list $\mathcal{L}$ in the Reveal query only when $\pi_{i, j}^{t}$ has been revealed and $d_{i, u}=a b P(u \in\{1, \cdots, n\})$, but $H_{2}\left(I D_{i}, I D_{j}, T_{i}^{t}, T_{j}^{t}, K_{1}^{t}, Z^{t}\right)$ had not been queried before the Reveal query. So, $S K_{i, j}^{t}$ has been randomly sampled.

$*$ Set $\zeta^{t}=S K_{i, j}^{t}$.

* Remove $\left(I D_{i}, I D_{j}, T_{i}^{t}, T_{j}^{t}, \Pi_{i, j}^{t}\right)$ from the list $\mathcal{L}$. Put $\left(I D_{i}, I D_{j}, T_{i}^{t}, T_{j}^{t}, K_{1}^{t}, Z^{t}, \zeta^{t}\right)$ into the list $H_{2}^{\text {list }}$.

* Return $S K_{i, j}^{t}$. 
- Otherwise, $\mathcal{B}$ chooses $\zeta^{t} \in\{0,1\}^{n}$ randomly, inserts $\left(I D_{i}, I D_{j}, T_{i}^{t}, T_{j}^{t}, K_{1}^{t}, Z^{t}, \zeta^{t}\right)$ into the list and returns $\zeta^{t}$.

- Otherwise, $\mathcal{B}$ chooses $\zeta^{t} \in\{0,1\}^{n}$ randomly, inserts $\left(I D_{i}, I D_{j}, T_{i}^{t}, T_{j}^{t}, K_{1}^{t}, Z^{t}, \zeta^{t}\right)$ into the list and returns $\zeta^{t}$.

- If a tuple indexed by $\left(I D_{i}, I D_{j}, T_{i}^{t}, T_{j}^{t}, K^{t}\right)$ is on the list, then $\mathcal{B}$ responds with $\zeta^{t}$.

- Otherwise, $\mathcal{B}$ chooses $\zeta^{t} \in\{0,1\}^{n}$ randomly, inserts $\left(I D_{i}, I D_{j}, T_{i}^{t}, T_{j}^{t}, K_{1}^{t}, Z^{t}, \zeta^{t}\right)$ into the list and returns $\zeta^{t}$.

Corrupt $\left(I D_{i}\right)$ queries: When receiving this query, $\mathcal{B}$ goes through list $H_{1}^{\text {list }}$. If $I D_{i, u}(u \in\{1, \cdots, n\})$ is not on the list, $\mathcal{B}$ queries $H_{1}\left(I D_{i, u}\right)$. $\mathcal{B}$ checks the value of $d_{i, u}$. If $d_{i, u}=\perp$, then $\mathcal{B}$ aborts the game (Event 1). Otherwise, $\mathcal{B}$ computes

$$
S_{i}=\sum_{u=1}^{n}\left(d_{i, u}+v_{i, u} y P\right)=\sum_{j=1}^{n}\left(l_{i, u} a P+v_{i, u} y P\right), R_{i}=\sum_{u=1}^{n} v_{i, u} P .
$$

$\mathcal{B}$ sends $d_{i}=\left(S_{i}, R_{i}\right)$ to $\mathcal{A}$.

Send $\left(\Pi_{i, j}^{t}, N\right)$ queries: $\mathcal{B}$ maintains a list $\Omega$ for each oracle of the form $\left(\Pi_{i, j}^{t}, \operatorname{tran}_{i, j}^{t}, r_{i, j}^{t}, K_{i j, 1}^{t}\right.$, $\left.K_{i j, 2}^{t}, S K_{i, j}^{t}\right)$. $\operatorname{tran}_{i, j}^{t}$ is the transcript of the oracle so far; $r_{i, j}^{t}$ is the random integer used by the oracle to generate the messages; $K_{i j, 1}^{t}, K_{i j, 2}^{t}$ and $S K_{i, j}^{t}$ are set $\perp$ initially. This list is updated in other queries as well. $\mathcal{B}$ proceeds in the following way:

- $\mathcal{B}$ looks through the list $H_{1}^{l i s t}$. If $j$ is not on the list, $\mathcal{B}$ queries $H_{1}\left(I D_{j, u}\right)$ where $1 \leq u \leq n_{j, i}^{t}$.

- $\mathcal{B}$ checks $t$.

- If $t=J, \mathcal{B}$ checks the values of $d_{j, u}$ and gives the different response depending on it as below.

* If there exists a tuple in $H_{1}^{l i s t}$ such that $d_{j, K}=\perp$ and $K \in\left\{1, \cdots, n_{j, i}^{t}\right\}$, then let $m_{1}=c P$. Compute

$$
m_{2}=-c \cdot y P=-y \cdot c P, \quad m_{3}=c \sum_{u=n_{j, i}^{t}+1}^{n} h_{j, u}=\sum_{u=n_{j, i}^{t}+1}^{n} l_{j, u} c P,
$$

where $l_{j, u}$ can be found from $H_{1}^{l i s t}$. Respond them along with $c P$ and set $r_{i, j}^{t}=\perp$.

* Otherwise, $\mathcal{B}$ aborts the game (Event 2).

- If $t \neq J, \mathcal{B}$ proceeds the protocol as follows.

* If all of $d_{i, u} \neq \perp$, randomly sample $r_{i, j}^{t} \in \mathbb{Z}_{q}^{*}$ and respond with

$$
\left(r_{i, j}^{t} P,-r_{i, j}^{t} y P, r_{i, j}^{t} \sum_{u=n_{j, i}^{t}}^{n} l_{j, u} P\right)
$$


* Otherwise, randomly sample $f_{i, j}^{t} \in \mathbb{Z}_{q}^{*}$ and respond with

$$
\left(f_{i, j}^{t} a P, f_{i, j}^{t} a y P,-f_{i, j}^{t} \sum_{u=n_{j, i}^{t}+1}^{n} l_{j, u} a P\right) .
$$

Reveal $\left(\Pi_{i, j}^{t}\right)$ queries: $\mathcal{B}$ maintains a list $\mathcal{L}$ with tuples of the form $\left(I D_{i}, I D_{j}, T_{i}, T_{j}, \Pi_{i, j}^{t}\right) . \mathcal{B}$ answers the queries as follows:

- Get the tuple of oracle $\Pi_{i, j}^{t}$ from $\Omega$.

- If oracle $\Pi_{i, j}^{t}$ has not accepted, respond with $\perp$.

- If $t=J$ or if the $J$-th oracle has been generated as $\Pi_{a, b}^{J}$ and $I D_{a}=I D_{j}, I D_{b}=I D_{i}$ and two oracles have the same session $I D$, abort the game (Event 3).

- If $S K_{i, j}^{t} \neq \perp$, return $S K_{i, j}^{t}$.

- Otherwise,

- If $r_{i, j} \neq \perp$, compute

$$
K_{i j, 1}=\hat{e}\left(S_{i}, m_{1}\right) \cdot \hat{e}\left(m_{2}, R_{i}\right) \cdot \hat{e}\left(m_{3}, a P\right) \cdot \hat{e}\left(\sum_{u=1}^{n_{j, i}^{t}} l_{j, u} P, a P\right)^{r_{i, j}^{t}},
$$

where $m_{1}, m_{2}$ and $m_{3}$ are the received messages on $\operatorname{tran}_{i, j}^{t}$. By making an $H_{2}$ query, set

$$
S K_{i, j}^{t}=H_{2}\left(I D_{i}, I D_{j}, T_{i}^{t}, T_{j}^{t}, K_{i j, 1}, r_{i, j}^{t} m_{1}\right),
$$

where

$$
T_{i}^{t}=\left(r_{i, j}^{t} P,-r_{i, j}^{t} y P, r_{i, j}^{t} \sum_{u=n_{j, i}^{t}+1}^{n} l_{j, u} P\right), \quad T_{j}^{t}=\left(m_{1}, m_{2}, m_{3}\right),
$$

if $\Pi_{i, j}^{t}$ is an initiator oracle, or $S K_{i, j}^{t}=H_{2}\left(I D_{j}, I D_{i}, T_{j}^{t}, T_{i}^{t}, K_{i j, 1}, r_{i, j}^{t} m_{1}\right)$ otherwise, and update $\Omega$ by putting $S K_{i, j}^{t}$ and return $S K_{i, j}^{t}$ as the response.

- Otherwise, it should have $d_{i, u}=a b P$ where $u=K$. Since $\mathcal{B}$ does not know $d_{i, K}$ and should not be able to compute $\hat{e}\left(m_{1}, a b P\right)$, thus should not be able to compute $K_{i j, 1}^{t}$. $\mathcal{B}$ proceeds as follows:

* Go though the list $H_{2}$ to find a tuple $\left(I D_{i}, I D_{j}, T_{i}^{t}, T_{j}^{t}, K_{i j, 1}^{t}, Z^{t}\right)$ if $I D_{i}$ is the initiator or a tuple $\left(I D_{j}, I D_{i}, T_{j}^{t}, T_{i}^{t}, K_{i j, 1}^{t}, Z^{t}\right)$ otherwise, meeting the equation $\hat{e}\left(f_{i, j}^{t} a P, m_{1}\right)=$ $\hat{e}\left(P, Z^{t}\right)$. If such $Z^{t}$ is found, then compute

$$
\begin{aligned}
K_{i j, 1}^{t}= & \hat{e}\left(S_{i}, m_{1}\right) \cdot \hat{e}\left(m_{2}, R_{i}\right) \cdot \hat{e}\left(m_{3},-P_{p u b}\right) \cdot \hat{e}\left(\sum_{j=1}^{n_{j, i}^{t}} h_{i, u}, a P\right)^{f_{i, j}^{t} a} \\
= & \hat{e}\left(\sum_{u=1, u \neq K}^{n}\left(l_{i, u} a P+v_{i, u} y P\right), m_{1}^{T_{j}^{t}}\right) \cdot \hat{e}\left(\frac{1}{f_{i, j}^{t}} Z_{t}, b P\right) \cdot \hat{e}\left(m_{2}, R_{i}\right) \cdot \hat{e}\left(m_{3},-P_{p u b}\right) \\
& \cdot \hat{e}\left(\sum_{j=1}^{n_{j, i}^{t}} l_{i, u} a P, a P\right)^{f_{i, j}^{t}}
\end{aligned}
$$


and set

$$
S K_{i, j}^{t}=H_{2}\left(I D_{i}, I D_{j}, T_{i}^{t}, T_{j}^{t}, K_{i j, 1}^{t}, Z^{t}\right)
$$

where

$$
T_{i}^{t}=\left(r_{i, j}^{t} P,-r_{i, j}^{t} y P, r_{i, j}^{t} \sum_{u=n_{j, i}^{t}+1}^{n} l_{j, u} P\right), \quad T_{j}^{t}=\left(m_{1}, m_{2}, m_{3}\right),
$$

if $\Pi_{i, j}^{t}$ is an initiator oracle, or $S K_{i, j}^{t}=H_{2}\left(I D_{j}, I D_{i}, T_{j}^{t}, T_{i}^{t}, K_{i j, 1}^{t}, Z^{t}\right)$ otherwise.

* Otherwise, randomly sample $S K_{i, j}^{t} \in\{0,1\}^{n}$, put $\left(I D_{i}, I D_{j}, T_{i}^{t}, T_{j}^{t}, \Pi_{i, j}^{t}\right)$ if $I D_{i}$ is the initiator or $\left(I D_{j}, I D_{i}, T_{j}^{t}, T_{i}^{t}, \Pi_{i, j}^{t}\right)$ into list $\mathcal{L}$.

* $\mathcal{B}$ returns $S K_{i, j}^{t}$ as the response and update $\Omega$ by putting $S K_{i, j}^{t}$.

Test $\left(\Pi_{i, j}^{t}\right)$ query: If $t \neq J$ or $\left(t=J\right.$ but) there is an oracle $\Pi_{j, i}^{s}$ which has the same session $I D$ as $\Pi_{i, j}^{t}$ that has been revealed, $\mathcal{B}$ aborts the game (Event 4 ). Otherwise, $\mathcal{B}$ responds to $\mathcal{A}$ a random number $\zeta \in\{0,1\}^{n}$.

After $\mathcal{A}$ finishes the queries, it returns its guess. Then $\mathcal{B}$ proceeds with the following steps:

Compute

$$
D=\hat{e}\left(S_{i}, m_{1}\right) \cdot \hat{e}\left(m_{2}, R_{i}\right) \cdot \hat{e}\left(m_{3}, a P\right),
$$

where $m_{1}, m_{2}$ and $m_{3}$ are the incoming messages. Also note that $S_{i}=\sum_{u=1}^{n} l_{i, u} a P+v_{i, u} y P$ and $R_{i}=\sum_{u=1}^{n} v_{i, u} P$ where $l_{i, u} \neq \perp$ and $v_{i, u} \neq \perp$ can be found from $H_{1}^{\text {list }}$ corresponding to identifier $I D_{i, u}$. Note that

$$
\begin{aligned}
K_{i, j}^{J} & =D \cdot \hat{e}\left(\sum_{u=1}^{n_{j, i}^{t}} Q_{j, u}, a P\right)^{c} \\
& =D \cdot \hat{e}\left(\sum_{u=1, u \neq K}^{n_{j, i}^{t}} l_{j, u} P, a P\right)^{c} \cdot \hat{e}(b P, a P)^{c} \\
& =D \cdot \hat{e}\left(\sum_{u=1, u \neq K}^{n_{j, i}^{t}} l_{j, u} c P, a P\right) \cdot \hat{e}(P, P)^{a b c} .
\end{aligned}
$$

$\mathcal{B}$ randomly samples $K_{l}$ from the $H_{2}^{\text {list }}$, and responds

$$
K_{l} /\left(D \cdot \hat{e}\left(\sum_{u=1, u \neq K}^{n_{j, i}^{t}} l_{j, u} c P, a P\right)\right)
$$

to the BDH challenge.

Claim 1 If $\mathcal{B}$ did not abort the game, $\mathcal{A}$ could not find inconsistence between the simulation and the real world. More precisely, if $\mathcal{A}$ noticed the inconsistence between the simulation and the real world when $\mathcal{B}$ did not abort the simulation, then the probability $\mathcal{B}$ solves the $B D H$ problem is non-negligible. 
Proof: $\mathcal{B}$ gives the satisficatory responses to most of the oracles by following the protocol specification honestly, except for the one $\Pi_{i, j}^{t}$ whose private key is $a b P$ and $H\left(I D_{i, k}\right)=b P$ and the incoming messages $\left(m_{1}, m_{2}, m_{3}\right)$ is from the tested oracle where $m_{1}=c P$. Note that the transcripts are one part of the input to $H_{2}$ which is modelled as the random oracle to compute the session key. If there is some difference between the reveal query on $\Pi_{i, j}^{t}$ and a query on $H_{2}$, it must have queried $H_{2}$ with $\Pi_{i, j}^{t}$ such that

$$
\begin{aligned}
K_{i j, 1}^{t} & =\hat{e}\left(S_{i}, m_{1}\right) \cdot \hat{e}\left(m_{2}, R_{2}\right) \cdot \hat{e}\left(m_{3},-a P\right) \cdot \hat{e}\left(\sum_{u=1}^{n_{j, i}^{t}} l_{j, u} P, a P\right)^{r_{i, j}^{t}} \\
& =\hat{e}\left(\sum_{u=1, u \neq K} l_{i, u} a P, c P\right) \cdot \hat{e}(a b P, c P) \cdot \hat{e}\left(m_{2}, R_{2}\right) \cdot \hat{e}\left(m_{3}, a P\right) \cdot \hat{e}\left(\sum_{u=1}^{n_{j, i}^{t}} l_{j, u} P, a P\right)^{r_{i, j}^{t}}
\end{aligned}
$$

If $\mathcal{A}$ can distinguish the session key $K_{i j, 1}^{t}$ in the simulation from the real world, then $\mathcal{B}$ can return

$$
\frac{K_{l}}{\hat{e}\left(\sum_{u=1, u \neq K} l_{i, u} a P, c P\right) \cdot \hat{e}\left(m_{2}, R_{2}\right) \cdot \hat{e}\left(m_{3},-a P\right) \cdot \hat{e}\left(\sum_{u=1}^{n_{j, i}^{t}} l_{j, u} P, a P\right)^{r_{i, j}^{t}}} .
$$

as the response to the $\mathrm{BDH}$ challenge with probability $\frac{1}{q_{2}}$, where $K_{l}$ is a random value choosing from $H_{2}$ by $\mathcal{B}$. This completes the proof.

Claim 2 During the simulation, the probability that $\mathcal{B}$ did not abort the game is non-negligible.

Proof: We now evaluate the probability that $\mathcal{B}$ did not abort during the game, i.e., Events 1 - 4 did not happen. $\mathcal{B}$ aborts the game only when at least one of following events happens:

1. Event 1 , denoted as $\mathcal{F}_{1}: \mathcal{A}$ corrupted party $i$ whose private key of $K$-th identity is represented by $\perp$, i.e., $\mathcal{A}$ made a query to party $i$ to get its private key if it chose $\Pi_{j, i}^{s}$ as the fresh oracle, which is disallowed according to the definition of the fresh oracle.

2. Event 2, denoted as $\mathcal{F}_{2}: \mathcal{A}$ impersonated party $i$ whose private key of $K$-th identity is represented by $\perp$ in the $s$-th session.

3. Event 3, denoted as $\mathcal{F}_{3}: \mathcal{A}$ revealed the $J$-th oracle or its partner oracle, which is against the definition of the fresh oracle.

4. Event 4 , denoted as $\mathcal{F}_{4}: \mathcal{A}$ did not choose the $J$-th oracle as the challenge fresh oracle or the parter of the fresh oracle has been revealed, which made that the test query can not work.

According to the rules of the game, we have

$$
\neg \mathcal{F}_{4} \wedge \neg \mathcal{F}_{2} \rightarrow \neg \mathcal{F}_{1}
$$

and

$$
\neg \mathcal{F}_{4} \rightarrow \neg \mathcal{F}_{3}
$$


Now, let $\mathcal{F}$ be the event that $\mathcal{B}$ did not abort during the game. Then, we get

$$
\begin{aligned}
\operatorname{Pr}[\mathcal{F}] & =\operatorname{Pr}\left[\neg \mathcal{F}_{1} \wedge \neg \mathcal{F}_{2} \wedge \neg \mathcal{F}_{3} \wedge \neg \mathcal{F}_{4}\right] \\
& =\operatorname{Pr}\left[\neg \mathcal{F}_{2} \wedge \neg \mathcal{F}_{4}\right] \\
& =\operatorname{Pr}\left[\neg \mathcal{F}_{2}\right] \cdot \operatorname{Pr}\left[\neg \mathcal{F}_{4}\right] \\
& \geq \frac{1}{q_{1}} \cdot \frac{1}{q_{o}} \\
& =\frac{1}{q_{1} \cdot q_{o}} .
\end{aligned}
$$

Claim 3 Let $\mathcal{H}$ be the event that $K=\hat{e}\left(S_{i}, m_{1}\right) \cdot \hat{e}\left(m_{2}, R_{i}\right) \cdot \hat{e}\left(m_{3},-a P\right) \cdot \hat{e}\left(\sum_{u=1}^{n_{j, i}^{t}} h_{i, u}, a P\right)^{c}$ was not queried on $\mathrm{H}_{2}$. Then $\operatorname{Pr}[\neg \mathcal{H}] \geq \epsilon$.

Proof:

It is easy to obtain

$$
\operatorname{Pr}[\mathcal{A} \text { wins } \mid \mathcal{H}]=\frac{1}{2}
$$

Since

$$
\begin{aligned}
\operatorname{Pr}[\mathcal{A} \text { wins }] & =\operatorname{Pr}[\mathcal{A} \text { wins } \mid \neg \mathcal{H}] \cdot \operatorname{Pr}[\neg \mathcal{H}]+\operatorname{Pr}[\mathcal{A} \text { wins } \mid \mathcal{H}] \cdot \operatorname{Pr}[\mathcal{H}] \\
& \leq \frac{1}{2} \operatorname{Pr}[\mathcal{H}]+\operatorname{Pr}[\neg \mathcal{H}] \\
& =\frac{1}{2} \operatorname{Pr}[\neg \mathcal{H}]+\frac{1}{2}
\end{aligned}
$$

and

$$
\begin{aligned}
\operatorname{Pr}[\mathcal{A} \text { wins }] & =\operatorname{Pr}[\mathcal{A} \text { wins } \mid \neg \mathcal{H}] \cdot \operatorname{Pr}[\neg \mathcal{H}]+\operatorname{Pr}[\mathcal{A} \text { wins } \mid \mathcal{H}] \cdot \operatorname{Pr}[\mathcal{H}] \\
& \geq \frac{1}{2} \operatorname{Pr}[\mathcal{H}] \\
& =\frac{1}{2}-\frac{1}{2} \operatorname{Pr}[\neg \mathcal{H}]
\end{aligned}
$$

So we have

$$
\operatorname{Pr}[\neg \mathcal{H}] \geq 2 \mid \operatorname{Pr}[\mathcal{A} \text { wins }]-\frac{1}{2} \mid=\epsilon
$$

Thus, the claim is correct.

Let $\mathcal{I}$ be the event that $\mathcal{B}$ found the correct $K_{l}$. Then combining all of the above results, we have

$$
\begin{aligned}
\operatorname{Pr}[\mathcal{B} \text { wins }] & =\operatorname{Pr}[\mathcal{F} \wedge \neg \mathcal{H} \wedge \mathcal{I}] \\
& \geq \frac{1}{q_{1} \cdot q_{o} \cdot q_{2}} \cdot \operatorname{Pr}[\neg \mathcal{H}] \\
& \geq \frac{1}{q_{1} \cdot q_{o} \cdot q_{2}} \cdot \epsilon,
\end{aligned}
$$

which contradicts to the hardness of the BDH problem.

This completes the security analysis of the protocol. 


\section{Conclusion}

We have proposed a novel dynamical identity-based authenticated key management protocol to optimize key management for a user with multiple identities. Our scheme allows a user with some basic identities to compute a new private key when some new identities are involved. We have proved our scheme in the random oracle model with the Bilinear Diffie-Hellman assumption. Our protocol has demonstrated obvious advantages in comparison with other schemes, especially for high efficiency in terms of communication, since it only needs constant-size number of messages for communication.

\section{Acknowledgements}

This work was supported by the National Natural Science Foundation of China (grant number 60973105, 90718017, 61170189), the Research Fund for the Doctoral Program of Higher Education (grant number 20111102130003) and the Fund of the State Key Laboratory of Software Development Environment (grant number SKLSDE-2011ZX-03, SKLSDE-2012ZX-11).

\section{References}

[1] Bellare M, Rogaway P. Entity authentication and key distribution. In: Advances in CryptologyCrypto'93, 1993; LNCS 773: 232-249.

[2] Blake-Wilson S, Johnson D, Menezes A. Key agreement protocols and their security analysis. In Proceedings of the sixth IMA International Conference on Cryptography and Coding, 1997; LNCS 1355: 30-45.

[3] Boneh D, Boyen X. Efficient selective-ID secure identity based encryption without random oracles. In Advances in Cryptology-EUROCRYPT'04, 2004; LNCS 3027: 223-238.

[4] Boneh D, Franklin M. Identity based encryption from the Weil pairing. In Proceedings of Advances in Cryptology-Crypto'01, 2001; LNCS 2139: 213-229.

[5] Boyen X. General Ad Hoc encryption from exponent inversion IBE. Advances in CryptologyEUROCRYPT'07 2007; LNCS 4515: 394-411.

[6] X. Cao, W. Kou, X. Du. A pairing-free identity-based authenticated key agreement protocol with minimal message exchanges. Information Sciences, 180(15), 2895-2903, 2010.

[7] Chen L, Cheng Z, Smart N.P. Identity-based key agreement protocols from pairings. International Journal Information Security 2007; 6: 213-241.

[8] Cheng Z, Nistazakis M, Comley R, Vasiu L. On the indistinguishability-based security model of key agreement protocols-simple cases. In Proceedings of ACNS 2004, 2004; June.

[9] Galbraith SD, Harrison K, Soldera D. Implementing the tate pairing. In: Proceedings of the 5th International Symposium on Algorithmic Number Theory-ANTS 02, 2002; LNCS 2369: 324-337. 
[10] Guo F, Mu Y, Chen Z. Identity-based encryption: how to decryot multiple ciphertexts using a single decryption key. In pairing 2007 2007; LNCS 4575: 392-406.

[11] Guo F, Mu Y, Chen Z, Xu L. Mluti-identity single-key decryption without random oracles. In Inscrypt 2007, 2007; LNCS 4990: 384-398.

[12] Guo H, Mu Y, Zhang X, Li Z. Authenticated Key Exchange Protocol with Selectable Identities. Wireless Communication and Mobile Computing 2011; 11(2): 226-239.

[13] Guo H, Mu Y, Li Z., Zhang X, Wu C., Teng J., An Efficient Dynamic Authenticated Key Exchange Protocol with Selectable Identities. Computers and Mathematics with Applications 2011; 61(9): 2518-2527.

[14] Menezes A, Okamoto T, Vanstone S.A. Reducing elliptic curve logarithms to logarithms in a finite field. IEEE Transactions on Information Theory 1993; 39(5): 1639-1646.

[15] Shamir A. Identity-based cryptosystems and signature schemes. In: Advances in CryptologyCrypto'84 1984; LNCS 196: 47-53.

[16] Sakai R, Kasahara M. ID based cryptosystems with pairing on elliptic curve. Cryptology ePrint Archive, Report 2003/054.

[17] Sakai R, Ohgishi K, Kasahara M. Cryptosystems based on pairing. In: Symposium on Cryptography and Information Security, 2000; Okinawa, Japan.

[18] L. Zhang, Q. Wu, B. Qin, et. al.. Provably secure one-round identity-based authenticated asymmetric group key agreement protocol. Information Sciences 2011; 181(19): 4318-4329. 